\title{
ABSTRACT GENERATION BASED ON RHETORICAL STRUCTURE EXTRACTION
}

\author{
Kenji Ono, Kazuo Sumita, Seiji Miike \\ Research and Development Center \\ Toshiba Corporation \\ Komukai-Toshiba-cho 1, Saiwai-ku, Kawasaki, 210, Japan \\ ono@isl.rdc.toshiba.co.jp
}

\section{ABSTRACT}

We have developed an automatic abstract generation system for Japanese expository writings bascd on rhetorical structure extraction. The system first extracts the rhetorical structure, the compound of the rhetorical relations between sentences, and then cuts out less important parts in the extracted structure to generate an abstract of the desired length. Evaluation of the generated abstract showed that it contains at maximum $74 \%$ of the most important sentences of the original text. The system is now utilized as a text browser for a prototypical interactive document retrieval system.

\section{INTRODUCTION}

Abstract generation is, like Machine Translation, one of the ultimate goal of Natural Language Processing. However, since conventional word-frequencybased abstract generation systems(e.g. [Kuhn 58]) are lacking in inter-sentential or discourse-structural analysis, they are liable to generate incolerent abstracts. On the other hand, conventional knowledge or script-based abstract generation systems(e.g. [Lehnert 80], [Fum 80]), owe their success to the limitation of the domain, and cannot be applied to document with varied subjects, such as popular scientific magazine. To realize a domain-independent abstract generation system, a computational theory for analyzing linguistic discourse structure and its practical procedure must be established.

Hobbs developed a theory in which he arranged three kinds of relationships between sentences from the text coherency viewpoint [Hobbs 79].

Grosz and Sidner proposed a theory which accounted for interactions between three notions on discourse: linguistic structure, intention, and attention [Grosz et al. 86].

litman and Allen clescribed a model in which a cliscourse structure of conversation was built by recognizing a participant's plans [Litman et al. 87]. These theories all depend on extra-linguistic knowledge, the accumulation of which presents a problem in the realization of a practical analyzer.

Cohen proposed a framework for analyzing the structure of argumentative discourse [Cohen 87], yet did not provide a concrete identification procedure for 'evidence' relationships between sentences, where no linguistic clues indicate the relationships. Also, since only relationships between successive sentences were considered, the scope which the relationships cover cannot be analyzed, even if explicit connectives are detected.

Mann and Thompson proposed a linguistic structure of text describing relationships between sentences and their relative importance [Mann et al. 87]. Ilowever, no method for extracting the relationships from superficial linguistic expressions was described in their paper.

We have developed a computational model of discourse for Japanese expository writings, and implemented a practical procedure for extracting discourse structure[Sumita 92]. In our model, discourse structure is defined as the rhetorical structure, i.e., the compound of rhetorical relations between sentences in text. Abstract generation is realized as a suitable application of the extracted rhetorical structure. In this paper we describe briefly our discourse model and discuss the abstract generation system based on it. 


\section{RHETORICAT STRUCTURE}

Rhetorical structure represents relations between various chunks of sentences in the body of each section. In this paper, the rhetorical structure is represented by two layers: intra-paragraph and inter-paragraph structures. An intra-paragraph structure is a structure whose representation units are sentences, and an inter-paragraph structure is a structure whose representation units are paragraphs.

In text, various rhetorical patterns are used to clarify the principle of argument. Among them, connective expressions, which state inter-sentence relationships, are the most significant. The typical grammatical categories of the connective expressions are connectives and sentence predicates. They can be divided into the thirty four categories which are excmplified in Table 1.

'Lable 1: Example of rhetorical relations

\begin{tabular}{|c|c|}
\hline Relation & Expressions \\
\hline serial ( $\langle\mathrm{SR}\rangle)$ & dakara (thus) \\
\hline $\begin{array}{l}\text { summarization } \\
(\langle\mathrm{SM}\rangle)\end{array}$ & kekkyoku (after all) \\
\hline negative $(\langle\mathrm{NG}\rangle)$ & shikashi (but) \\
\hline example ( $\langle\mathrm{EG}\rangle)$ & tatocba (for exanuple) \\
\hline especial $(\langle\mathrm{ES}\rangle)$ & tokuni (particularly) \\
\hline reason ( $\langle R S\rangle$ ) & nazenara (because) \\
\hline supplement (〈SP & mochiron (of colurse) \\
\hline background ( $\angle B I\rangle)$ & juurai (hitherto) \\
\hline parallel $(\langle\mathrm{PA}\rangle)$ & mata (and) \\
\hline extension $(\langle\mathrm{EX}\rangle)$ & kore wa (this is) \\
\hline rephrase $(\langle R F\rangle)$ & tsumari (that is to say) \\
\hline direction $(\langle\mathrm{D} I\rangle)$ & $\begin{array}{l}\text { bokode wa ... wo noberu } \\
\text { (here ... is described) }\end{array}$ \\
\hline
\end{tabular}

The rhetorical relation of a sentence, which is the relationship to the preceding part of the text, can be extracted in accordance with the connective expression in the sentence. For a sentence without any explicit connective expressions, extension relation is set to the sentence. The relations exemplified in rable 1 are used for representing the rhetorical structure.

Pig. 1 shows a paragraph from an article titled "A Zero-Crossing late Which Estimates the Frequency of a Speech Signal," where underlined words indicate connective expressions. Although the fourtl and fifth sentences are clearly the exemplification of the first three sentences, the sixth is not. Also the sixth sentence is the concluding sentence for the first five. 'I'hus, the rhetorical structure for this text can be represented by a binary-tree as shown in lig. 2.This structure is also represented as follows:

$[[[1\langle\mathrm{EX}\rangle 2]\langle\mathrm{ES}\rangle[3\langle\mathrm{EG}\rangle[1\langle\mathrm{EX}\rangle \quad 5]]]\langle\mathrm{SR}\rangle \quad 6]$

1: In the context of discrete-time signals, zerocrossing is said to occur if successive samples have different algebraic signs.

2: The rate at which zero crossings occur is a simple measure of the frequency content of a signal.

3 : This is particularly true of narrow band signals.

4: For example, a sinusoidal signal of frequency $F_{0}$, sampled at a rate $F_{s}$, has $F_{4} / H_{s}$ samples per cycle of the sine wave.

5: Wach cycle hats two zero crossings so that the long-term average rate of zero-crossings is $Z=2 F_{0} / F_{s}$.

6: Tlius, the average zero-crossing rate gives a reasonable way to estimate the frequency of a sine wave.

(I.R.Mabiner and R.W.Schafer, Digital Pracessing of Speech Signals, Prentice-IIall, 1978, p.127.)

Figure 1: Text example

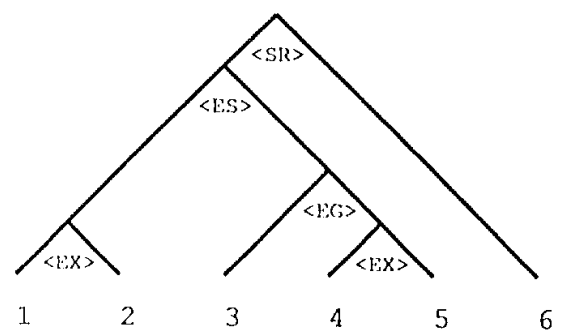

Figure 2: Thetorical structure for the lext in Fig.l

The rhetorical structure is represented by a binary tree on the analogy of a syntactic tree of a natural language sentence. Each sub tree of the rhetorical structure forms an argumentative constituent, just as cach sub-tree of the syntaclic tree forms a grammatical constituent. Also, a sub-tree of the rhetorical structure is sub-categorized by a relation of its parent node as well as a syntactic tree. 


\section{RHETORICAL STRUCTURE EX- TRACTION}

The rhetorical structure represents logical relations between sentences or blocks of sentences of each section of the document. A rhetorical structure analysis determines logical relations between sentences based on linguistic clues, such as connectives, anaphoric expressions, and idiomatic expressions in the input text, and then recognizes an argumentative chunk of sentences.

Rhetorical structure extraction consists of six major sub-processes:

(1) Sentence analysis accomplishes morphological and syntactic analysis for each sentence.

(2) Rhetorical relation extraction detects rhetorical relations and constructs the sequence of sentence identifiers and relations.

(3) Segmentation detects rhetorical expressions between distant sentences which define rhetorical structure. They are added onto the sexpence produced in step 2, and form restrictions for generating structures in step 4. For example, expressions like “...3 reasons. First, ... Second, ... Third, ...", and "... Of course, ... ...But, ..." are extracted and the structural constraint is added onto the sequence so as to form a chunk between the expressions.

(4) Candidate generation generates all possible rhetorical structures described by binary trees which do not violate segmentalion restrictions.

(5) Preference judgement selects the structure candidate with the lowest penalty score, a value determined based on preference rules on every two neighboring relations in the candiclate. This process selects the structure candidate with the lowest penalty score, a value determined based on preference rules on every two neighboring relations in the candidate. A preference rule used in this process represents a heuristic local preference on consecutive rhetorical relations between sentences. Consicler the sequence $[\mathrm{P}\langle\mathrm{EG}\rangle \mathrm{Q}\langle\mathrm{SR}\rangle \mathrm{R}$ ], where $\mathrm{P}, \mathrm{Q}, \mathrm{R}$ are arbitrary (blocks of) sentences. The premise of $R$ is obvously not only $Q$ but both $P$ and $Q$. Since the discussion in $\mathrm{P}$ and $\mathrm{Q}$ is considered to close locally, structure [ $[P\langle E G\rangle Q]\langle S R\rangle R]$ is preferable to $[P\langle E G\rangle[Q\langle S R\rangle R]]$. Penally scores are imposed on the structure candidates violating the preference rules, for example, for the text in Fig. 1, the structure candiclates which contain the sulsstructure

[3 $\langle E G\rangle[[4\langle E X>5]<S R>6]]$, which says sentence six is the entailment of sentence four and five only, are penalized. The authors have investigated all pairs of rhelorical relations and derived those preference rules.

The system analyzes inter-paragraph structures after the analysis of intra-paragraph structures. While the system uses the rhetorical relations of the first sentence of each paragraph for this analysis, it executes the sane steps as it cloes for the intra- paragraph analysis.

\section{ABSTRACT GENERATION}

The system generates the abstract of each section of the document by examining its rhetorical structure. The process consists of the following 2 stages.

\section{(1) Sentence evaluation \\ (2) Structure reduction}

In the sentence etaluation stage, the system calculate the importance of each sentence in the original text based on the relative importance of rhetorical relations. They are categorized into three types as shown in Table 2. For the relations categorized into RightNucleus, the right node is more important, from the point of view of abstract generation, than the left node. In the case of the LeftNucleus relations, the situation is vice versa. And both nodes of the BothNucleus relations are equivalent in their importance. for example, since the right node of the serial relation (e.g., yotte (thus)) is the conclusion of the left node, the relation is categorized into RightNucleus, and the right node is more important than the left node.

The Actual sentence cvaluation is carried out in a demerit marking way. In order to determine important text segments, the system imposes penalties on both nodes for each rhetorical relation according to its relative importance. The system imposes a penalty on the left node for the RightNucleus relation, and also on the right node for the Left Nucleus relation. It adds penalties from the root node to the terminal nodes in turn, to calculate the penalties of all norles.

'Then, in the structure reduction stage, the system recursively cuts out the nodes, from the terminal nodes, which are imposed the highest penalty. The list of terminal nodes of the final structure becomes an abstract for the original document. Suppose that the abstract is longer than the expected length. In 
that case the system cuts out terminal nodes from the last sentences, which are given the same penalty score.

If the text is written loosely, the rhetorical structure generally contains many BothNucleus relations (e.g., parallel(mata(and, also)), and the system cannot gradate the penalties and cannot reduce sentences smoothly.

After sentences of each paragraph are reduced, inter-paragraph structure reduction is carried out in the same way based on the relative importance jud gement on the inter-paragraph rhetorical structure.

If the penalty calculation mentioned above is accomplished for the rhetorical structure shown in Fig. 2, each penalty score is calculated as shown in Fig. 3. In Fig. 3 italic numbers are the penalties the system imposed on each node of the structure, and broken lines are the boundary between the nodes inposed different penalty scores. The figure shows that sentence four and five have penalty score three, that sentence three has two, that sentence one and two have one, and that sentence six has no penalty score. In this case, the system selects sentence onc, two, three and six for the longest abstract, and and also could select sentence one, two and six as a shorter abstract, and also could select sentence six as a still more shorter abstract.

After the sentences to be included in the albstract are determined, the system alternately arranges the sentences and the connectives from which the relations were extracted, and realizes the text of the abstract.

The important feature of the generated abstracts is that since they are composed of the rhetoricaly consistent units which consist of several sentences and form a rhetorical substructure, the abstract does not contain fragmentary sentences which cannot be understood alone. For example, in the abstract generation mentioned above, sentence two does not appear solely in the abstract, but appears always with sentence one. If sentence two appeared alone in the abstract without sentence one, it would be difficult to understand the text.

\section{EVALUATION}

The generated abstracts were evaluated from the point of view of key sentence coverage. 30 cditorial articles of "Asahi Shinbun", a Japanese newspaper, and 12 technical papers of "Toshiba Review", a journal of Toshiba Corp. which publishes short expository papers of three or four pages, were selected and three subjects judged the key sentences and the most important key sentence of each text. As for the edito-
Table 2: Relative importance of rhetorical relations

\begin{tabular}{|c|l|c|}
\hline \hline Relation Type & \multicolumn{1}{|c|}{ Relation } & Import. Node \\
\hline Right Nucleus & $\begin{array}{l}\text { serial, } \\
\text { summarizar } \\
\text { tion, } \\
\text { negative, ... }\end{array}$ & right node \\
\hline Left Nucleus & $\begin{array}{l}\text { example, } \\
\text { reason, } \\
\text { especial, } \\
\text { supplement, } \\
\text { B. }\end{array}$ & left node \\
\hline BothNucleus & $\begin{array}{l}\text { parallel, } \\
\text { extension, } \\
\text { rephrase, ... }\end{array}$ & both nodes \\
\hline
\end{tabular}

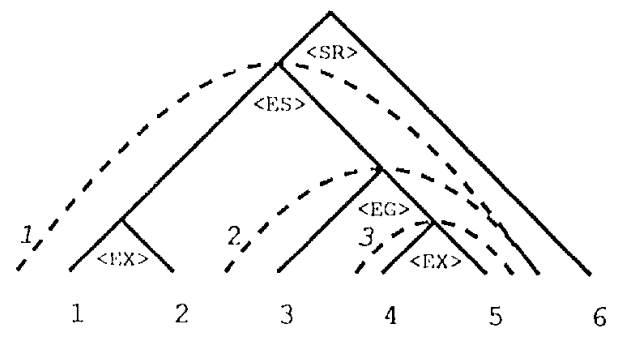

Figure 3: Penalties on relative importance for the rhetorical structure in Fig. 2

rial articles, The average correspondence rates of the key sentence and the most important key sentence among the subjects were $60 \%$ and $60 \%$ respectively. As for the technical papers, they were $60 \%$ and $80 \%$ respectively.

Then the alstracts were generaled and were compared with the selected ley sentences. The result is shown in Thable 3. As for the technical papers, the average length ratio( abstract/original) was 21 $\%$, and the coverage of the key sentence and the rnost important key sentence were $51 \%$ and $74 \%$ respectively. Whereas, as for the editorials, the average length ratio(alsstract/original) was $30 \%$, and the coverage of the key sentence and the most important key sentence were $41 \%$ and $60 \%$ respectively.

The rcason why the compression rate and the key sentence coverage of the teclnical papers were higher than that of the editorials is considered as follows. The technical papers contains so many rhetorical expressions in general as to be expository. 
That is, they provide many linguistic clues and the system can extract the rhetorical structure exactly. Accordingly, the structure can be reduced further and the length of the abstract gets shorter, without omitting key sentences. On the other hand, in the editorials most of the relations between sentences are supposed to be understood semantically, and are not expressed rhetorically. Therefore, they lack linguistic clues and the system cannot extract the rhetorical structure exactly.

Table 3: Key sentence coverage of the abstracts

\begin{tabular}{|c|c|c|c|c|}
\hline Material & total & length & \multicolumn{2}{|c|}{ cover ratio } \\
\cline { 3 - 5 } & num. & ratio & $\begin{array}{c}\text { key } \\
\text { sentence }\end{array}$ & $\begin{array}{c}\text { most } \\
\text { important } \\
\text { sentence }\end{array}$ \\
\hline $\begin{array}{c}\text { (A sahi Shinbun) } \\
\text { tech, journal } \\
(\text { Toshiba Review) }\end{array}$ & 42 & 0.24 & 0.51 & 0.74 \\
\hline
\end{tabular}

\section{CONCLUSION}

We have developed an automatic abstract generation system for Japancse expository writings based on rhetorical structure extraction.

The rhetorical structure provides a natural order of importance among sentences in the text, and can be used to determine which sentence should be extracted in the abstract, accordling to the desired length of the abstract. The rhetorical structure also provides the rhetorical relation between the extracted sentences, and can be used to generate appropriate connectives between them.

Abstract generation based on rhetorical structure extraction has four merits. First, unlike conventional word-frequency-based abstract generation systems(e.g. [Kuhn 58]), the generated alsstract is consistent with the original text in that the connectives between sentences in the abstract reflect their relation in the original text. Second, once the rhetorical structure is obtained, various lengths of generated abstracts can be generated easily. This can be done by simply repeating the reduction process until one gets the desired length of abstract. 'Third, unlike conventional knowledge or script-based abstract generation systems(e.g. [Lehnert 80], [Fum 86]), the rhetorical structure extraction does not need prepared knowledge or scripts related to the original text, and can be used for texts of any domain, so long as they contain enough rhetorical expressions to be expository writings. Fourth, the generated abstract is composed of rhetoricaly consistent units which consist of several sentences and form a rhetorical substructure. so the abstract does not contain fragmentary sentences which cannot be unclerstood alone.

The limitations of the system are mainly due to errors in the rhetorical structure analysis and the sentence-selection-type abstract generation. the evaluation of the accuracy of the rhetorical structure analysis carried out previously ( [Sumita 92]) showed $74 \%$. Also, to make the length of the abstract shorter, It is necessary to utilize an inner-sentence analysis and to realize a phrase-selection-type abstract generation based on it. The anaphora-resolution and the topic-supplementation must also be realized in the analysis.

The system is now utilized as a text browser for a prototypical interactive document retrieval system.

\section{References}

[Cohen 87] Cohen, R. : "Analyzing the Structure of Argumentative Discourse", Computalional Linguistics, Vol.13, pp.11-24, 1987.

[Fum 86] Fum, D. : "Tailoring Importance Evaluation to Reader's Goals: A Contribution to Descriptive'Text Summarization", Proc. of Coling, pp.252-259, 1986.

[Cirosz et al, 86] Grosz, B.J. and Sidner, C.L. : "Attention, Intentions and the Structure of Discourse", Computational Linguistics, Vol.12, pp.175-204, 1986.

[Hoblss 79] Hobbs, J.R.: "Coherence and Coreference", Cognilive Science, Vol.3, 1979, pp.67-90.

[Kuhn 58] Kulun, II.P.: "The Automatic Creation of Literature Abstracts", IBM Journal, Apr. 1958, pp.159105.

[Leluner 80] Lelnert, W. : Naurative Text Summarization", Proc of AAAl, pP.337-339, 1980.

[Litman et al. 87] Litman, D.J. and Allen, J.F.: "A Plan Recognition Model for Subdialogues in Conversations", Cognilive Science, Vol.11, 1987, pp.163-200.

[Mann et al. 87] Mann, W.C. and Thompson, S.A. : "Rhetorical Structure Theory: A Framework for the Analysis of Texts", USC/Information Science Institule Research Report RR-87-190, 1987.

[Sumita 92] Sumita, K., et al. : " $A$ Discourse Structure Analyzer for Japanese Text", Proc. Int. Conf. Fifth Generation Computer Systems 1992 (FGCS'92), p). 1133-1140, 1902 